\title{
An improved method to build lung cancer PDX models by surgical resection samples and its association with $\mathrm{B} 7-\mathrm{H} 3$ expression
}

\author{
Yuxuan Wang", Biao Zhang", Haitao Huang, Tingjing Wang \\ Department of Thoracic Surgery, The First Affiliated Hospital of Soochow University, Suzhou 215006, China \\ Contributions: (I) Conception and design: T Wang; (II) Administrative support: T Wang, H Huang; (III) Provision of study materials or patients: Y \\ Wang, B Zhang; (IV) Collection and assembly of data: Y Wang, B Zhang; (V) Data analysis and interpretation: Y Wang; (VI) Manuscript writing: All \\ authors; (VII) Final approval of manuscript: All authors. \\ "These authors contributed equally to this work. \\ Correspondence to: Tingjing Wang. Department of Thoracic Surgery, The First Affiliated Hospital of Soochow University, 899 Pinghai Road, Suzhou \\ 215006, China. Email: tingjing0410@126.com.
}

Background: Patient-derived xenograft (PDX) models are biologically stable and can accurately reflect the primary tumor in histopathology and genetic expression in many cancers. In lung cancer, the models' engraftment rate by endobronchial ultrasound transbronchial needle aspiration (EBUS-TBNA) and computed tomography (CT)-guided biopsy is exceptionally low, and this limits the development of PDX models in lung cancer research. In this study, we aimed to improve the traditional method, and explore the feasibility and convenience of establishing lung cancer PDX models using the samples directly from surgical resection. We also endeavored to explore the correlation between PDX formation and primary tumor B7-H3 protein expression.

Methods: From September 2017 to July 2018, 24 patients were enrolled in this study. The pathological diagnoses were as follows: 15 adenocarcinomas, 7 squamous cell carcinomas, 1 large cell carcinoma, and 1 small cell carcinoma. The tumor tissues were cut into sizes of $2 \times 2 \times 2 \mathrm{~mm}^{3} /$ fragment and inoculated subcutaneously into immunodeficiency mice with a trocar needle. The engrafted tumors were passaged at least 3 generations, and B7-H3 IHC staining was performed on primary tumors. To explore the correlation between PDX formation and $\mathrm{B} 7-\mathrm{H} 3$ protein expression, we also performed $\mathrm{H} \& \mathrm{E}$ staining to evaluate whether the established PDX models could retain the histological features of the primary tumor.

Results: Xenograft formation success was achieved in 19 out of 24 cases $(79.2 \%)$. The time between implantation to tumor formation was an average of 81.5 days (27-154 days) in P1, an average of 44.4 days (14-122 days) in $\mathrm{P} 2$, and an average of 26.9 days (12-75 days) in P3. The diameter of the tumor was associated with tumor engraftment: the longer the diameter, the easier engraftment formation was $(\mathrm{P}=0.0342)$. The data also suggests that lung cancers with B7-H3 expression greatly induced PDX formation $(\mathrm{P}=0.0375)$. The histological characters of each passage resembled the primary tumors.

Conclusions: This study shows that the samples from carefully selected lung cancer by surgical resection can be used for the establishment of PDX models with a high success rate; this improved method is closer to actual clinical work. Compared with the same kind of research, the success rate of the xenograft indicates significant improvement, so this improved method is suitable for promotion and application. The results of H\&E staining showed that the histological characters of each passage resembled the primary tumors. Furthermore, the diameter of the tumor was associated with tumor engraftment, and higher B7-H3 expression demonstrated a statistically significant difference compared with the PDX model formation.

Keywords: B7-H3; non-small cell lung cancer (NSCLC); patient-derived tumor xenograft (PDX); pre-clinical model

Submitted Jun 26, 2019. Accepted for publication Oct 17, 2019.

doi: $10.21037 /$ tcr.2019.10.40

View this article at: http://dx.doi.org/10.21037/tcr.2019.10.40 


\section{Introduction}

Today, lung cancer, especially non-small cell lung cancer (NSCLC), has become one of the most common tumors in the world (1). The therapeutic regimen of lung cancer includes surgical resection, chemotherapy, radiotherapy, targeted therapy, and immunotherapy. The preclinical model is indispensable for cancer research, and the use of this model is a core component in every aspect of translational cancer research, ranging from the biological understanding of the disease to the development of new treatments. Over the past 40 years, the NCI-60 cancer cell line panel has been the best-characterized and most frequently used collection of human cancer models used for in vitro drug screening and development. Although these cell lines are convenient and easy to use, they lack predictive value with regards to activity in specific cancer types in clinical trials (2). Patient-derived tumor xenograft (PDX) models have become the preferred animal model in cancer research, preclinical cancer research, high-throughput drug screening, and assessment. In the current research, PDX models are biologically stable and can accurately reflect the primary tumor in histopathology and genetic expression (3). Using the samples from endobronchial ultrasound transbronchial needle aspiration (EBUS-TBNA) and computed tomography (CT)-guided biopsy, the success rate of model formation in lung cancer ranges from $26 \%$ to $47.1 \%$. This low rate of successful implantation has limited the PDX models' application.

Surgery is the best available treatment in NSCLC stage I to III. From surgical resection, we can acquire sufficient samples to establish the PDX models. In this study, we improved the traditional method and evaluated the feasibility of establishing PDX models using surgical resection samples. Thus, PDX models remain the preferred method to understanding many cancers and drug responses in pre-clinical research $(4,5)$.

The B7 family, an important family of co-stimulatory molecules, has been shown to play a critical role in $\mathrm{T}$ cell activation (6,7). B7-H3, a newly identified member of the B7 family, has recently gained attention in the field. It was identified in 2001 by database searches of a human dendritic cell (DC)-derived cDNA library (8). Its mRNA was found to be widely expressed in normal human tissues, but the B7-H3 protein was rarely expressed in normal organs (6). It has been reported that $\mathrm{B} 7-\mathrm{H} 3$ protein was overexpressed in a wide variety of solid tumors, including lung cancer, breast cancer, renal cancer, ovarian cancer, colorectal cancer, and hepatocellular cancer (9-14).

In NSCLC, the expression of B7-H3 was positively correlated with the number of tumor-infiltrating lymphocytes, and the higher expression of $\mathrm{B} 7-\mathrm{H} 3$, the higher the probability that patients would develop lymph node metastasis, and the worse the prognosis would be in NSCLC patients (9). As a tumor-associated antigen, its tumor immunological function is still controversial, which may be related to the activation status of $\mathrm{T}$ cells (15). Except for its immunological function, B7-H3 also plays a non-immunological role in cancer progression (16). It may be involved in tumor invasion, recurrence, and metastasis. Studies have found that B7-H3 can promote cell epithelialmesenchymal transition (EMT) through the JAK2-STAT3 pathway, which further promotes the invasion, recurrence, and metastasis of tumor cells $(17,18)$. By silencing the B7-H3 gene of tumor cells, the invasion and migration ability of tumor cells is significantly reduced (17). High expression of $\mathrm{B} 7-\mathrm{H} 3$ tends to predict high aggressiveness and poor clinical prognosis of tumors $(12,19)$. In this study, immunodeficient mice were used as the host to avoid the interference of the immunological function of $\mathrm{B} 7-\mathrm{H} 3$, and to explore whether the $\mathrm{B} 7-\mathrm{H} 3$ expression level of primary tumors would affect the success rate of modeling.

Different strains of mice and different inoculation sites will lead to different modeling success rates. Taking NSCLC as an example, Fichtner et al. (20) transplanted fresh specimens into NOD/SCID mice subcutaneously, and the success rate was only $25 \%$. However, by inoculating under the renal capsule into NOD/SCID mice, Dong et al. (21) reported a modeling success rate of up to $90 \%$. Nakajima $e t$ al. (22) subcutaneously inoculated the samples into NSG mice, with a success rate of $42 \%$.

The engraftment rate of PDX models will directly affect the cost of the experiment, the length of the experimental period, and the clinical value of pharmacodynamic tests. Meanwhile, the lower rate will also affect the development of precision medicine and individualized treatment. We hope that this study can help improve and optimize the traditional implantation method, make the modeling process closer to clinical practice, simplify the operation process, increase the success rate of the modeling as much as possible, and do so while controlling costs. We also analyzed the relationship between the success rate and clinical data, including patients' gender, age, tumor size, lymph node metastasis, TNM stage, etc. $\mathrm{B} 7-\mathrm{H} 3$ protein expression intensity in primary tumors was analyzed using immunohistochemistry (IHC) staining, analysis of the relationship between B7-H3 expression level, 
and engraftment rate. The established models in this study also serve as a carrier for subsequent pathogenesis, targeted therapy, and drug efficacy tests of NSCLC.

\section{Methods}

\section{Patients and tumor samples}

In this study, the first passage of PDX models was created from tumor specimens of treatment-naive patients-all patients who were diagnosed with lung cancer or were suspicious for lung cancer. All the specimens were cut directly from the surgery performed by the First Affiliated Hospital of Soochow University, and the diagnosis was certified on fast icy cross-section during the operation.

\section{Establishment of patient-derived tumor xenografts in immune-defective mice}

Fresh tumor specimens were maintained in Custodiol $^{\circledR}$ HTK-Solution in the refrigerator with the temperature was set at $4^{\circ} \mathrm{C}$, and the implantation was performed within $24 \mathrm{~h}$ after surgery. The tumor tissues were cut into sizes of $2 \times 2 \times 2 \mathrm{~mm}^{3} /$ fragment and then inoculated subcutaneously into 6-8-week-old NPG/Vst female mice with a trocar needle. This mouse strain was used in the initial xenograft passage to provide optimal engraftment conditions in the absence of mature lymphocytes and NK cells. Once tumors were engrafted, the following generation PDX models were supported by the NOD-SCID mice to achieve stable model establishment. Tumor growth was measured twice a week using a Vernier caliper. The tumor-bearing mice were sacrificed, and the tumors were removed when the tumors grew up to $750 \mathrm{~mm}^{3}$ or if the health of the mice was compromised. The tumor tissues were separated and sliced into several pieces, some for serial passage into new mouse hosts as described above, the others stained with $\mathrm{H} \& \mathrm{E}$ staining for histological review and cryopreservation at $-80^{\circ} \mathrm{C}$ for further analyses. Engrafted tumors were passaged for at least 3 generations (called P1, P2, P3, and so on). All procedures were performed under sterile conditions at the SPF facility. All interventions and animal care procedures were performed following the Guidelines and Policies for Animal Surgery provided by our hospital and were approved by the Institutional Animal Use and Care Committee.

\section{Immunobistochemistry}

Immunohistochemistry was performed as follows: $4-\mu \mathrm{m}$ thick consecutive sections were cut by a microtome, dewaxed in xylene, and rehydrated through graded ethanol solutions. Antigens were retrieved by heating the tissue sections at $100{ }^{\circ} \mathrm{C}$ for $30 \mathrm{~min}$ in the EDTA solution. Sections were cooled down and immersed in $0.3 \% \mathrm{H}_{2} \mathrm{O}_{2}$ solution for 20 min to block endogenous peroxidase activity, and then rinsed in PBS for $5 \mathrm{~min}$, blocked with 5\% BSA at room temperature for $20 \mathrm{~min}$, and incubated with primary antibodies against $\mathrm{B} 7-\mathrm{H} 3$ at $4{ }^{\circ} \mathrm{C}$ overnight. Negative controls were performed by replacing the specific primary antibody with PBS. Following a PBS wash, sections were incubated with secondary antibodies for $30 \mathrm{~min}$ at room temperature. Diaminobenzene was used as the chromogen, and hematoxylin was used as the nuclear counterstain. Sections were dehydrated, cleared, and mounted.

B7-H3 positive reactions were defined as those showing brown signals in the cell cytoplasm. A staining index (value, $0-12$ ) was determined by multiplying the score for staining intensity with the score for the positive area. The intensity was scored as follows: 0 , negative; 1 , weak; 2 , moderate; and 3, strong. The frequency of positive cells was defined as follows: 0 , no positive cell; 1 , less than $10 \% ; 2,11 \%$ to $50 \% ; 3,51 \%$ to $80 \%$; 4 , greater than $80 \%$.

For statistical analysis, scores of 0 to 3 were considered weakly positive, scores of 4 to 8 were considered moderately positive, and scores of 9 to 12 were considered as a strong expression.

\section{HÆ̈E staining}

H\&E staining was performed as follows: $4-\mu \mathrm{m}$ thick consecutive sections were cut by a microtome, dewaxed in xylene, and rehydrated through graded ethanol solutions. the slide was immersed for $30 \mathrm{~s}$ with agitation by hand in $\mathrm{H}_{2} \mathrm{O}$, then dipped into a Coplin jar containing Mayer's hematoxylin and agitate for $30 \mathrm{~s}$, and rinsed with $\mathrm{H}_{2} \mathrm{O}$ for $1 \mathrm{~min}$. The slide was then stained with a $1 \%$ eosin $\mathrm{Y}$ solution for $30 \mathrm{~s}$ with agitation. The sections were dehydrated with two changes of $95 \%$ alcohol and two changes of $100 \%$ alcohol for 30 s each. The alcohol was extracted with two changes of xylene. One drop of mounting medium was added and covered with a coverslip.

\section{Statistical analysis}

Statistical analysis was performed using GraphPad Prism software 5.0 (La Jolla, CA, USA). Statistical significance of differences between groups was determined using Fisher's 
exact test or two-tailed Student's $t$-test; a $\mathrm{P}$ value less than 0.05 was considered significant.

\section{Results}

\section{Tumor formation rate and time}

From September 2017 to June 2018, 24 patients were enrolled in this study. The baseline characteristics of patients are summarized in Table 1. The pathological diagnoses were as follows: 15 adenocarcinomas, 7 squamous cell carcinomas, 1 large cell carcinoma, and 1 small cell carcinoma. The median patient age was 61 years, and $58.3 \%$ were male. Sixteen $(66.7 \%)$ patients were diagnosed as stage I/II, and the other patients were diagnosed as stage III (33.3\%). There were 12 patients (50\%) with lymph node metastasis. Xenograft formation success was achieved in 19 out of 24 cases $(79.2 \%)$. The time between implantation to tumor formation was an average of 81.5 days (27-154 days) in $\mathrm{P} 1$, an average of 44.4 days (14-122 days) in $\mathrm{P} 2$, and an average of 26.9 days in P3, as shown in Figure 1 and Table 2. All engrafted tumors were serially passaged at least 3 times.

We found that SCC had a higher implantation rate than ADC, and advanced stage (stage III) lung cancer had a higher implantation rate than a tumor in the early stage (stage I \& II). Nevertheless, the difference among these groups was not statistically significant $(\mathrm{P}=0.3057-0.3636)$ (Table 3). The diameter of the tumor was associated with tumor engraftment: the longer the diameter, the easier the engraftment formation was $(\mathrm{P}=0.0342)$ (Figure 2).

\section{PDX model growth curve}

The daily behavior of animals was observed twice a week. After the tumor was formed, the tumor diameter was measured weekly by Vernier caliper, and the tumor volume was calculated with the formula above. We found that from P1 to P3, the tumorigenesis time of the tumor was gradually shortened, and the shortening trend was apparent. The tumor volume growth curve was plotted as shown in Figure 3.

\section{Relationship between B7-H3 expression of primary tumor and success rate of modeling}

The B7-H3 protein level in patients was measured with IHC, and the score was valued (Figure 4A). Among these 24 cases of primary tumors, 12 were detected with B7-H3 strong expression $(+++), 5$ with moderately positive $(++)$ and 2 with weakly positive (+), as shown in (Figure 4). The data suggests that the success rate of PDX models is closely related to the expression intensity of lung cancer $\mathrm{B} 7-\mathrm{H} 3$ protein $(\mathrm{P}=0.0375)$.

\section{Histological features between primary tumors and PDX models}

We also performed $\mathrm{H} \& \mathrm{E}$ staining to evaluate whether PDX models retained the histological features of the primary tumor. The results of H\&E staining showed that the histological characters of each passage resembled the primary tumors (Figure 5).

\section{Discussion}

The long modeling time and low success rate of PDX models limit a larger scale of its application. Many researchers have reported that the success rate of PDX model engraftment in lung cancer is from $26 \%$ to $47.1 \%$ (17-19,22-25). The tumor samples are obtained from surgical resection or EBUS-TBNA or CT-guiding biopsy, but in our study, the success rate was up to 79.2\% (19/24). The previous PDX modeling scheme was analyzed, and the features of this program are described below.

\section{Advantages}

(I) Instead of NOD/SCID mice or nude mice, NPG/ Vst mice were used for the first passage. NPG/Vst mice were combined with severe immunodeficiency, which had missed T lymphocytes, B lymphocytes, and NK cells, and had little rejection of the allograft, so they were more prone to tumor formation. NOD/ SCID mice were used in subsequent subcultures; this inoculation strategy can effectively improve the growth rate of the $\mathrm{P} 1$ tumor and can also save experimental funds.

(II) We used the samples directly from surgical resection, while most other studies used the samples from EBUS-TBNA or CT-guide biopsy. Although EBUSTBNA and CT-guide biopsy technology has the advantages of minimal trauma and rapid recovery, its available sample size is too small, thus, it is difficult to guarantee a sufficient number of tumor cells inoculated in immunocompromised mice.

(III) Due to the particularity of the operation department, 
Table 1 Clinical characteristics of enrolled patients

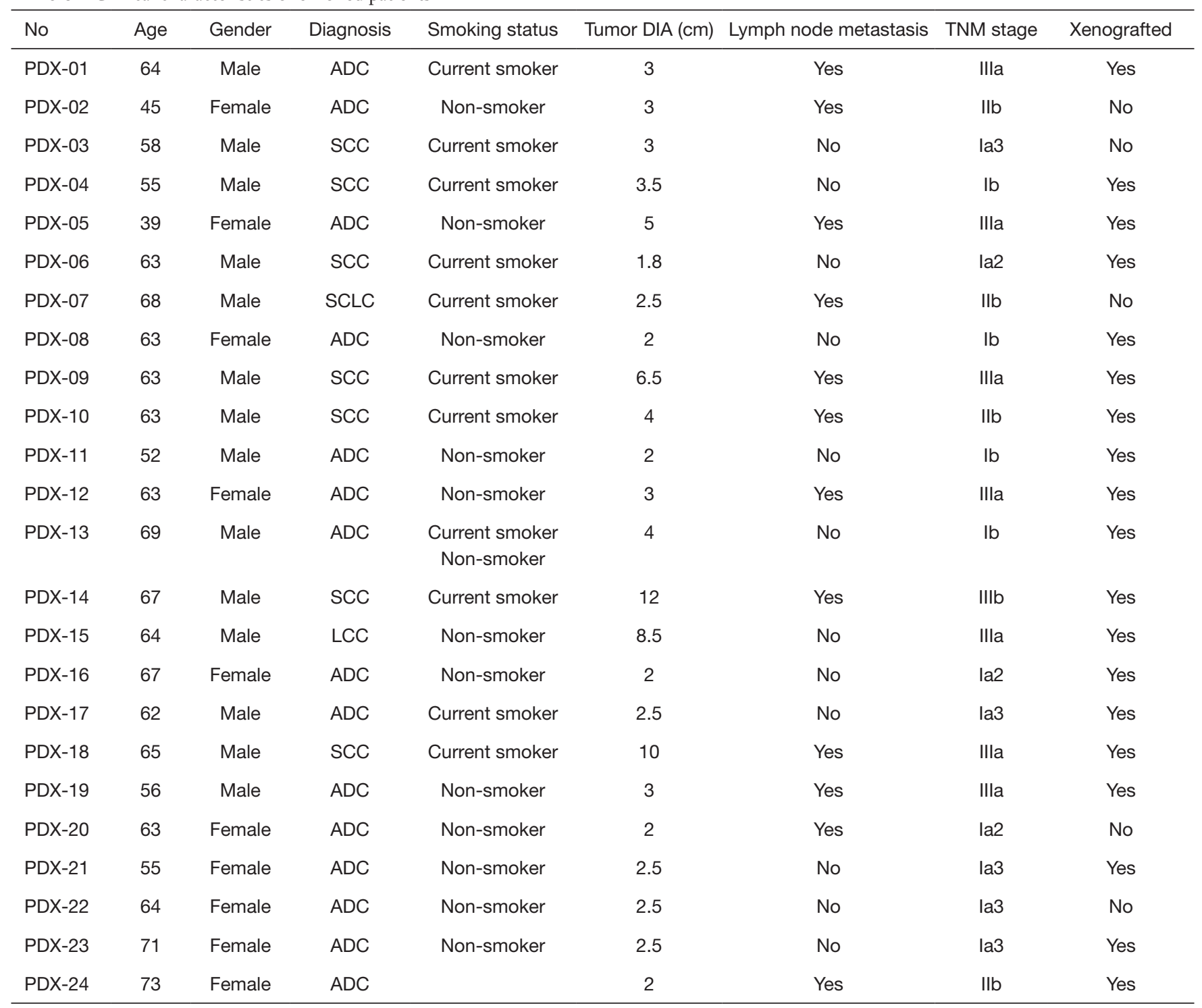

and in order not to interrupt the mice $12 \mathrm{~h} / 12 \mathrm{~h}$ patterns of life, we can't guarantee to inoculate the samples immediately after the operation, so we preserved the samples in $4{ }^{\circ} \mathrm{C}$ HTK solution and completed the procedure by the next morning at the latest. The results showed that this method does not affect the engraftment rate of PDX modeling.

(IV) We selected patients with tumor diameter $\geq 2 \mathrm{~cm}$ by chest CT scan. Larger tumor diameter means more active growth of tumor cells, shorter doubling time, and more samples available, which ensures that enough samples were inoculated into the mice.
(V) The PDX models of SCC established in this study and its primary cultured cells have been successfully applied to the study of relevant targets of SCC, and the results of these two models can be mutually confirmed (26).

\section{Disadvantages}

(I) PDX models based on surgically resected samples are not suitable for patients without surgical indications and those who cannot tolerate or refuse surgery due to their physical conditions. PDX modeling for these 


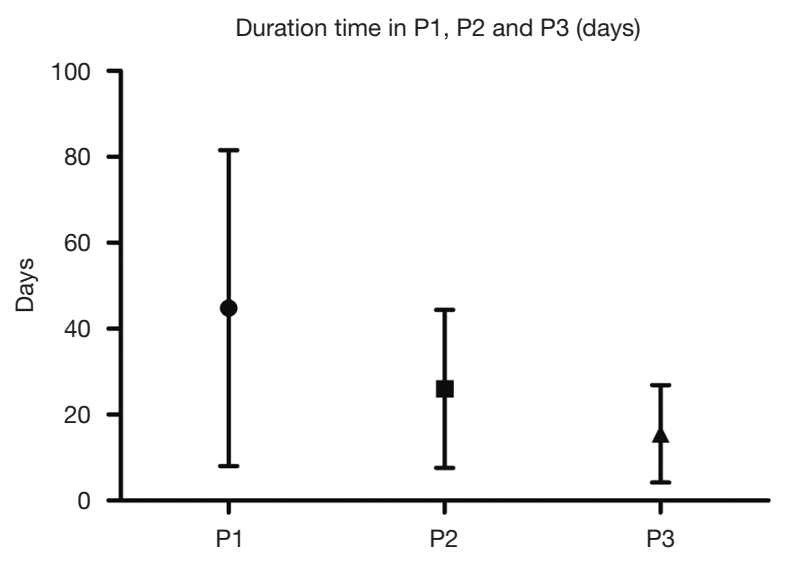

Figure 1 Duration time in P1, P2, and P3 (days). The duration time after samples inoculated subcutaneously into NPG/Vst female mice to tumor formation (with a volume of at least $500 \mathrm{~mm}^{3}$ ).

Table 2 Duration for tumor engraftment in P1, P2 and P3 (days)

\begin{tabular}{|c|c|c|c|}
\hline No. & $\mathrm{P} 1$ & P2 & P3 \\
\hline PDX-01 & 88 & 24 & 18 \\
\hline PDX-04 & 46 & 39 & 30 \\
\hline PDX-05 & 80 & 64 & 17 \\
\hline PDX-06 & 27 & 17 & 13 \\
\hline PDX-08 & 154 & 122 & 72 \\
\hline PDX-09 & 123 & 14 & 10 \\
\hline PDX-10 & 66 & 21 & 40 \\
\hline PDX-11 & 117 & 34 & 21 \\
\hline PDX-12 & 72 & 65 & 42 \\
\hline PDX-13 & 60 & 32 & 24 \\
\hline PDX-14 & 51 & 36 & 18 \\
\hline PDX-15 & 103 & 27 & 15 \\
\hline PDX-16 & 100 & 21 & 14 \\
\hline PDX-17 & 103 & 96 & 26 \\
\hline PDX-18 & 53 & 36 & 22 \\
\hline PDX-19 & 38 & 31 & 21 \\
\hline PDX-21 & 89 & 26 & 21 \\
\hline PDX-23 & 46 & 21 & 12 \\
\hline PDX-24 & 132 & 118 & 75 \\
\hline Average & 81.5 & 44.4 & 26.9 \\
\hline
\end{tabular}

Table 3 Clinical features of 24 lung cancer patients with PDX establishment

\begin{tabular}{|c|c|c|c|c|}
\hline Parameters & Xenografted & Un-xenografted & $\chi^{2}$ & $P$ value \\
\hline Gender & & & 0.8734 & 0.3500 \\
\hline Male & 12 & 2 & & \\
\hline Female & 7 & 3 & & \\
\hline Diagnosis & & & 3.618 & 0.3057 \\
\hline ADC & 12 & 3 & & \\
\hline SCC & 6 & 1 & & \\
\hline SCLC & 0 & 1 & & \\
\hline LCC & 1 & 0 & & \\
\hline Smoke & & & 0.0866 & 0.7686 \\
\hline Yes & 9 & 2 & & \\
\hline No & 10 & 3 & & \\
\hline \multicolumn{2}{|c|}{ Lymph node metastasis } & & 0.2526 & 0.6152 \\
\hline Yes & 10 & 2 & & \\
\hline No & 9 & 3 & & \\
\hline TNM stage & & & 0.8250 & 0.3636 \\
\hline $1 / I I$ & 11 & 5 & & \\
\hline III & 8 & 0 & & \\
\hline
\end{tabular}

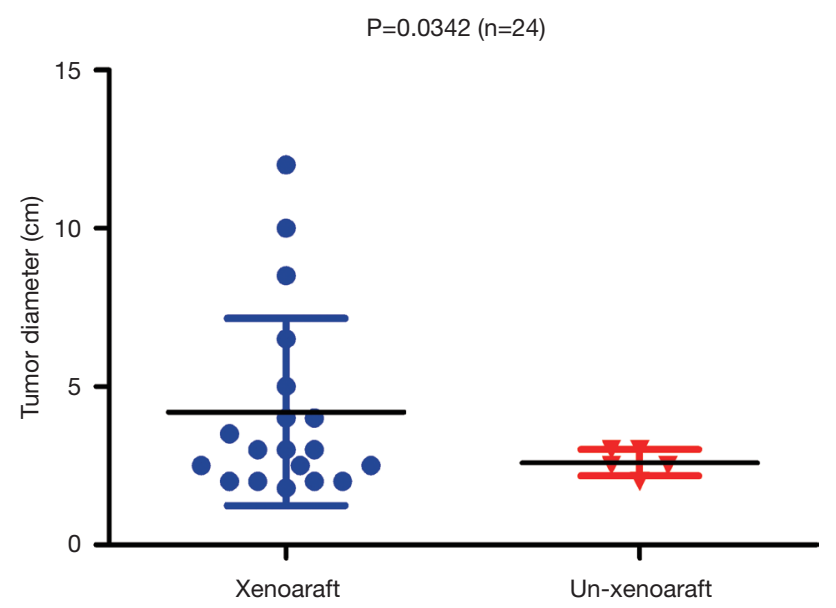

Figure 2 The diameter of the tumor was associated with tumor engraftment: the longer the diameter, the easier engraftment formation was $(\mathrm{P}=0.0342)$. 

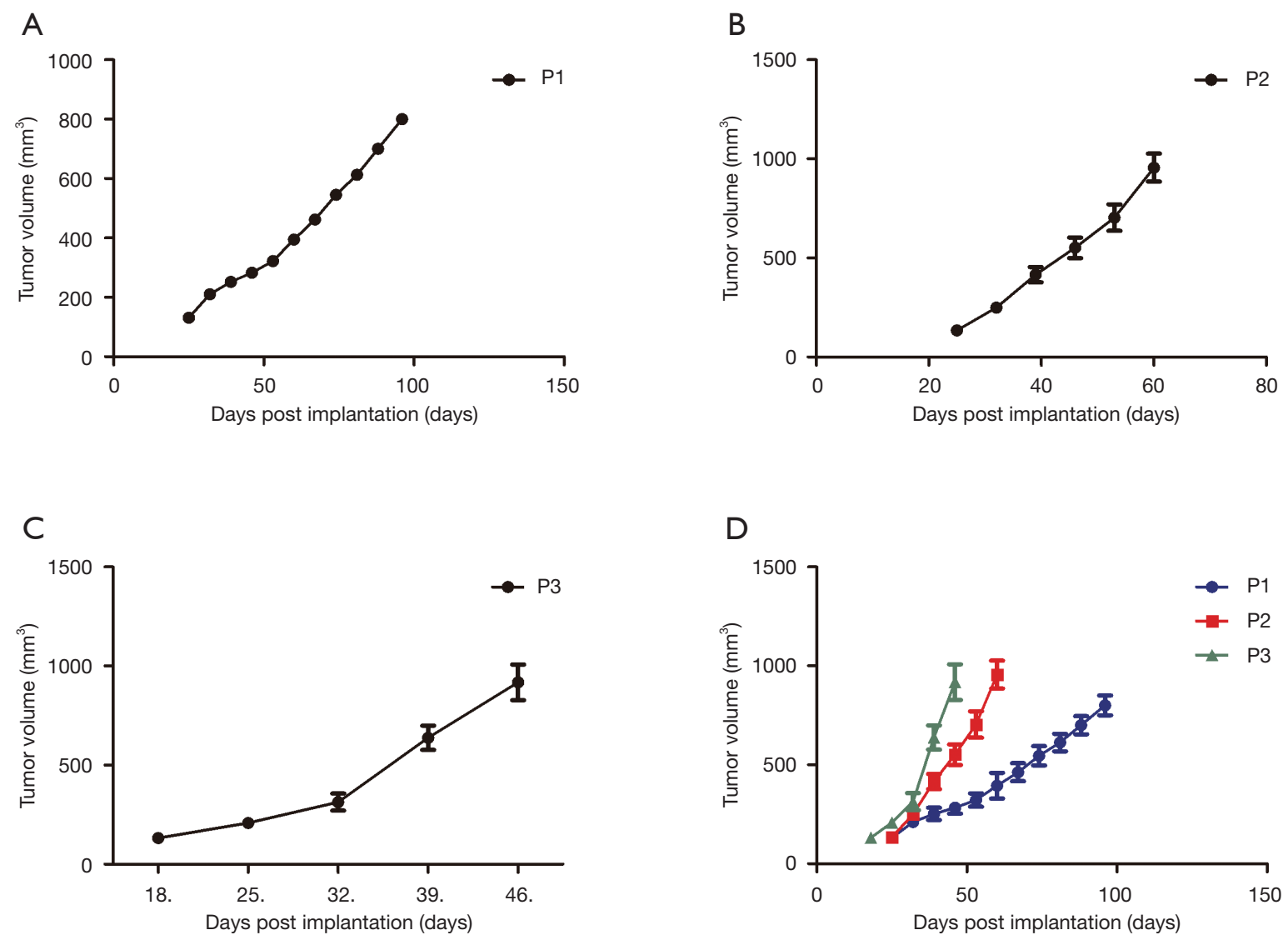

Figure 3 PDX model growth curve. The plotted tumor volume growth curve from passage 1 to passage 3 .

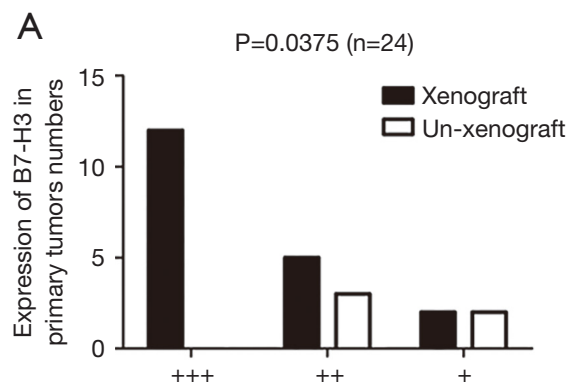

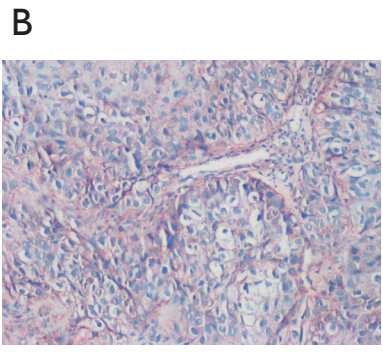

Strongly postive

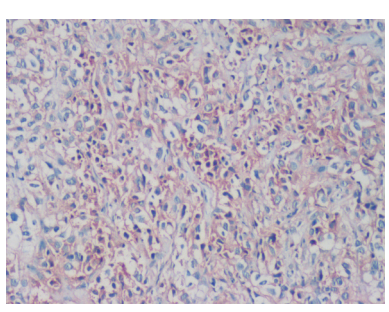

Moderately postive

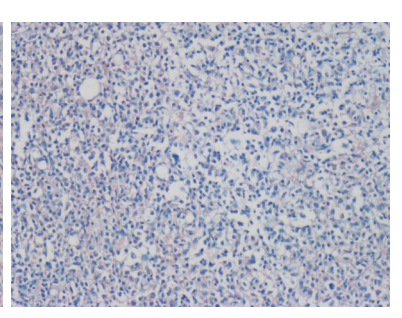

Weakly postive

Figure 4 The expression of B7-H3 in primary tumor with IHC. (A) The lung cancers with B7-H3 expression greatly induced PDX formation $(\mathrm{P}<0.05)$ (Fisher's exact test); (B) the expression of $\mathrm{B} 7-\mathrm{H} 3$ protein levels measured with IHC $(\times 10)$. Strongly positive $(\mathrm{PDX}-14)$, moderately positive (PDX-10), weakly positive (PDX-06).

patients still relies on exhaled pleural effusion cells, EBUS-TBNA, and CT-guided biopsy to obtain samples.

(II) Although subcutaneous implantation is the most commonly used method for the establishment of PDX models, compared with orthotopic transplantation, it cannot simulate the microenvironment of tumor growth at its best, but the orthotopic transplantation model for lung cancer is rarely studied due to the difficulty in operation and the tumor volume measurement, which is also the direction of our future research. 
PT

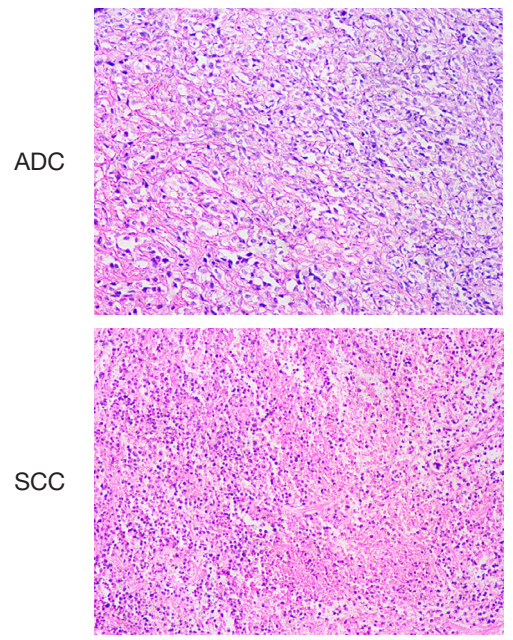

P1



P2

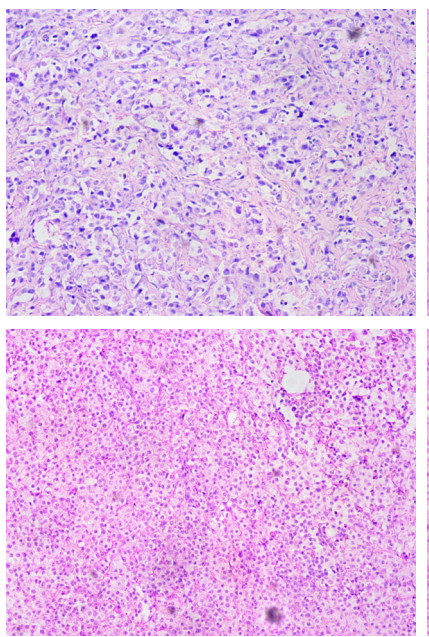

P3

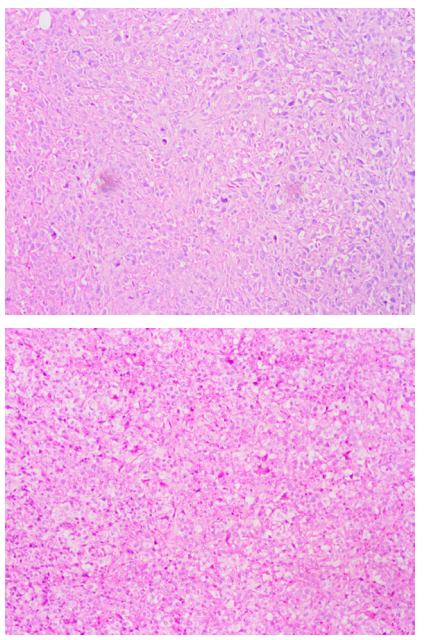

Figure 5 The H\&E staining in primary tumor, P1, P2, and P3 $(\times 10)$. The H\&E staining of primary tumor $(\mathrm{PT})$, passage $1(\mathrm{P} 1)$, passage 2 (P2), and passage 3 in SCC (PDX-14) and ADC (PDX-17). The histological characters of each passage resembled the primary tumors.

The only small cell lung cancer sample in this study was not successful in PDX establishment. Due to the low incidence of small cell lung cancer and the lack of surgical indications in patients with advanced small cell lung cancer, it is challenging to obtain surgical specimens, which requires more follow-up studies.

For quite some time, cancer-cell-line-derived tumor xenografts $(\mathrm{CDX})$ have been contributing significantly to the identification and testing of many chemotherapy drugs. Compared to the PDX models, CDX models have been shown to have numerous shortcomings, including the lack of tumor heterogeneity and microenvironments, and thus CDX models cannot reflect human cancer types accurately (27). PDX models are widely used in cancer research, especially in pre-clinical cancer research, and PDX has become the golden standard model in clinical drug response $(28,29)$.

Statistical analysis of the relationship between the clinical data and the tumorigenesis rate showed that the tumorigenesis rate was not related to the patients' age, TNM stage, or lymph node metastasis, but was positively correlated with the tumor diameters. Few cases were enrolled in this study, and postoperative follow-up was not conducted for the patients. The relationship between the success rate, the length of tumor doubling time, and the survival of the patients is not yet known. Statistical analysis will be made after increasing the sample size.

Tumor diameter was an essential item in the TNM stage. The $8^{\text {th }}$ TNM for NSCLC was significantly different from that in the 7 th edition in terms of the $\mathrm{T}$ stage, which was sufficient to demonstrate the crucial role of tumor diameter in tumor progression. Microvascular infiltration (MVI) is a powerful predictor of poor prognosis in NSCLC patients. Shimada et al. (30). showed that the occurrence frequency of MVI varied with tumor diameter, in the groups with tumor diameter $<2 \mathrm{~cm}, 2-3 \mathrm{~cm}$, and $>3 \sim 5 \mathrm{~cm}$, there was a significant difference in the effect of MVI positivity on survival rate $(\mathrm{P}<0.05)$. For patients with MVI $(+)$ in the above groups, the proportion of 5 -year relapse-free interphase decreased with the increase of tumor diameter.

This study also found that the expression intensity of primary tumor $\mathrm{B} 7-\mathrm{H} 3$ was also closely related to the success rate of PDX modeling. As a costimulatory molecule, the receptor of $\mathrm{B} 7-\mathrm{H} 3$ is not known, and its immunological function has remained controversial. However, its non-immunological function has been recognized to include promoting tumor cell adhesion, invasion, and migration $(15,31)$.

In this study, immunodeficient mice were used as the host to avoid the interference of the immunological function of $\mathrm{B} 7-\mathrm{H} 3$, and the results confirmed that the primary tumor B7-H3 expression intensity was closely related to the success rate of PDX modeling.

Several other studies have shown that B7-H3 can promote tumor cell adhesion and invasion, and our study indicates that the tumors with high $\mathrm{B} 7-\mathrm{H} 3$ expression have a higher success rate in model engraftment formation. 


\section{Conclusions}

(I) In this study, the traditional PDX modeling method was improved, making the modeling method closer to the actual work of surgical clinics. The success rate of modeling was significantly improved compared with similar studies, and the effect and cost performance were considered in the selection of immunodeficient mice. The application of PDX models for the individualized and precise treatment of lung cancer patients is more feasible when it is compared to other models.

(II) The H\&E staining result shows that compared to their corresponding primary tumors, the histological characteristics of PDX models from P1 to P3 were well maintained.

(III) The success rate of PDX modeling is closely related to the diameter of the primary tumor, and the primary tumor B7-H3 expression intensity is positively correlated with the success rate of modeling.

\section{Acknowledgments}

The authors would like to thank GenePharma (Suzhou) for providing the SPF-class housing of the laboratory, and we would also like to express our thanks to Ms. Zhangyan Zhong for technical support of the implantation.

Funding: This work was supported by the National Natural Science Foundation of China (Grant no. 31300746 , 81672281), and the Jiangsu Science and Technology Project (grant no. BE2015638).

\section{Footnote}

Conflicts of Interest: All authors have completed the ICMJE uniform disclosure form (available at http://dx.doi. org/10.21037/tcr.2019.10.40). The authors have no conflicts of interest to declare.

Etbical Statement: The authors are accountable for all aspects of the work in ensuring that questions related to the accuracy or integrity of any part of the work are appropriately investigated and resolved. All procedures performed in studies involving human participants were in accordance with the ethical standards of the institutional and/or national research committee(s) and with the Helsinki Declaration (as revised in 2013). Individual informed consent was waived due to the retrospective nature of the study. All interventions and animal care procedures were performed following the Guidelines and Policies for Animal Surgery provided by our hospital and were approved by the Institutional Animal Use and Care Committee.

Open Access Statement: This is an Open Access article distributed in accordance with the Creative Commons Attribution-NonCommercial-NoDerivs 4.0 International License (CC BY-NC-ND 4.0), which permits the noncommercial replication and distribution of the article with the strict proviso that no changes or edits are made and the original work is properly cited (including links to both the formal publication through the relevant DOI and the license). See: https://creativecommons.org/licenses/by-nc-nd/4.0/.

\section{References}

1. Bray F, Ferlay J, Soerjomataram I, et al. Global cancer statistics 2018: GLOBOCAN estimates of incidence and mortality worldwide for 36 cancers in 185 countries. CA Cancer J Clin 2018;68:394-424.

2. Hausser HJ, Brenner RE. Phenotypic instability of Saos-2 cells in long-term culture. Biochem Biophys Res Commun 2005;333:216-22.

3. Kuracha MR, Thomas P, Loggie BW, et al. Patientderived xenograft mouse models of pseudomyxoma peritonei recapitulate the human inflammatory tumor microenvironment. Cancer Med 2016;5:711-9.

4. Tentler JJ, Tan AC, Weekes CD, et al. Patient-derived tumour xenografts as models for oncology drug development. Nat Rev Clin Oncol 2012;9:338-50.

5. Siolas D, Hannon GJ. Patient-derived tumor xenografts: transforming clinical samples into mouse models. Cancer Res 2013;73:5315-9.

6. Greenwald RJ, Freeman GJ, Sharpe AH. The B7 family revisited, Annu Rev Immunol 2005;23:515-48.

7. Yoshinaga SK, Whoriskey JS, Khare SD, et al. T-cell co-stimulation through B7RP-1 and ICOS. Nature 1999;402:827-32.

8. Chapoval AI, Ni J, Lau JS, et al. B7-H3: a costimulatory molecule for T cell activation and IFN-gamma production. Nat Immunol 2001;2:269-74.

9. Sun Y, Wang Y, Zhao J, et al. B7-H3 and B7-H4 expression in non-small-cell lung cancer. Lung Cancer 2006;53:143-51.

10. Fauci JM, Straughn JM, Ferrone S, et al. A review of $\mathrm{B} 7-\mathrm{H} 3$ and $\mathrm{B} 7-\mathrm{H} 4$ immune molecules and their role in ovarian cancer. Gynecol Oncol 2012;127:420-5.

11. Ingebrigtsen VA, Boye K, Tekle C, et al. B7-H3 expression 
in colorectal cancer: nuclear localization strongly predicts poor outcome in colon cancer. Int J Cancer 2012;131:2528-36.

12. Flies DB, Han X, Higuchi T, et al. Coinhibitory receptor $\mathrm{PD}-1 \mathrm{H}$ preferentially suppresses $\mathrm{CD}^{+} \mathrm{T}$ cell-mediated immunity. J Clin Invest 2014;124:1966-75.

13. Liu T, Huo Y, Li G, et al. A negative correlation between B7-H3 expression and the number of CD8+ T cell infiltration in primary hepatocellular carcinoma tissues. $\mathrm{Xi}$ Bao Yu Fen Zi Mian Yi Xue Za Zhi 2014;30:1291-4.

14. Wang G, Wu Z, Wang Y, et al. Therapy to target renal cell carcinoma using 131I-labeled B7-H3 monoclonal antibody. Oncotarget 2016;7:24888-98.

15. Kang FB, Wang L, Jia HC, et al. B7-H3 promotes aggression and invasion of hepatocellular carcinoma by targeting epithelial-to-mesenchymal transition via JAK2/STAT3/Slug signaling pathway. Cancer Cell Int 2015; $15: 45$.

16. Wang L, Kang FB, Shan BE. B7-H3-mediated tumor immunology: Friend or foe. Int J Cancer 2014;134:2764-71.

17. Merk J, Rolff J, Becker M, et al. Patient-derived xenografts of non-small-cell lung cancer: a pre-clinical model to evaluate adjuvant chemotherapy. Eur J Cardiothorac Surg 2009;36:454-9.

18. Hao C, Wang L, Peng S, et al. Gene mutations in primary tumors and corresponding patient-derived xenografts derived from non-small cell lung cancer. Cancer Lett 2015;357:179-85.

19. Stewart EL, Mascaux C, Pham NA, et al. Clinical Utility of Patient-Derived Xenografts to Determine Biomarkers of Prognosis and Map Resistance Pathways in EGFR-Mutant Lung Adenocarcinoma. J Clin Oncol 2015;33:2472-80.

20. Fichtner I, Rolff J, Soong R, et al. Establishment of patient-derived non-small cell lung cancer xenografts as models for the identification of predictive biomarkers. Clin Cancer Res 2008;14:6456-68.

21. Dong X, Guan J, English JC, et al. Patient-derived first generation xenografts of non-small cell lung

Cite this article as: Wang Y, Zhang B, Huang H, Wang T. An improved method to build lung cancer PDX models by surgical resection samples and its association with $\mathrm{B} 7-\mathrm{H} 3$ expression. Transl Cancer Res 2019;8(8):2848-2857. doi: 10.21037/ tcr.2019.10.40 cancers: promising tools for predicting drug responses for personalized chemotherapy. Clin Cancer Res 2010;16:1442-51.

22. Nakajima T, Geddie W, Anayama T, et al. Patient-derived tumor xenograft models established from samples obtained by endobronchial ultrasound-guided transbronchial needle aspiration. Lung Cancer 2015;89:110-4.

23. Fu S, Zhao J, Bai H, et al. High-fidelity of non-small cell lung cancer xenograft models derived from bronchoscopyguided biopsies. Thorac Cancer 2016;7:100-10.

24. Ma Y, Zhang P, An G, et al. Induction of PatientDerived Xenograft Formation and Clinical Significance of Programmed Cell Death Ligand 1 (PD-L1) in Lung Cancer Patients. Med Sci Monit 2016;22:4017-25.

25. Roife D, Kang Y, Wang L, et al. Generation of patientderived xenografts from fine needle aspirates or core needle biopsy. Surgery 2017;161:1246-54.

26. Huang H, Wang Y, Li Q, et al. miR-140-3p functions as a tumor suppressor in squamous cell lung cancer by regulating BRD9. Cancer Lett 2019;446:81-89.

27. Bousquet G, Janin A. Patient-Derived Xenograft: An Adjuvant Technology for the Treatment of Metastatic Disease. Pathobiology 2016;83:170-6.

28. Julien S, Merino-Trigo A, Lacroix L, et al. Characterization of a large panel of patient-derived tumor xenografts representing the clinical heterogeneity of human colorectal cancer. Clin Cancer Res 2012;18:5314-28.

29. Weroha SJ, Becker MA, Enderica-Gonzalez S, et al. Tumorgrafts as in vivo surrogates for women with ovarian cancer. Clin Cancer Res 2014;20:1288-97.

30. Shimada Y, Saji H, Kato Y, et al. The Frequency and Prognostic Impact of Pathological Microscopic Vascular Invasion According to Tumor Size in Non-Small Cell Lung Cancer. Chest 2016;149:775-85.

31. Chen YW, Tekle C, Fodstad O. The immunoregulatory protein human $\mathrm{B} 7 \mathrm{H} 3$ is a tumor-associated antigen that regulates tumor cell migration and invasion. Curr Cancer Drug Targets 2008;8:404-13. 